\title{
An Enhanced Q Algorithm Based on EPC-C1G2 RFID Protocol
}

\author{
Cunliang Niu, Hao Zhang ${ }^{*}$ and Tao Lin \\ School of Computer Science and Engineering, Hebei University of Technology, Tianjin, 300401, China \\ ${ }^{*}$ Corresponding author
}

\begin{abstract}
For the low system efficiency of RFID anti-collision $Q$ algorithm which was proposed by ECP-C1G2, we propose an enhanced $Q$ algorithm that embeds BS(Binary Splitting) algorithm into $Q$ algorithm. The collision tags will be identified by $B S$ algorithm in the process of $Q$ Algorithm. Simulation results show that, the proposed algorithm significantly reduces the collision slots, total slots, and improves the identification efficiency of system. The identification efficiency of system remains around 0.4 .
\end{abstract}

Keywords-RFID anti-collision; Q algorithm; EPC-C1G2; tags; identification efficiency of system

\section{INTRODUCTION}

The existing RFID anti-collision algorithms can be divided into two categories: ALOHA-based algorithms ${ }^{[1]}$ and tree-based algorithms ${ }^{22]}$. ALOHA-based algorithm is widely used because of its fast recognition and easy implementation. EPC-C1G2 standard ${ }^{[3]}$ proposed anti-collision Q algorithm is also based on DFSA(Dynamic Frame Slotted ALOHA) algorithm. Q algorithm is an adaptive frame length adjustment algorithm. But Q algorithm no longer performs tag estimation operations, this causes the error between the frame length and the tag quantity to be bigger, system identification efficiency of Q algorithm is only about $0.34^{[4]}$.

Considering the low system identification efficiency of Q algorithm, many scholars have proposed some improved algorithms. In [5], a scheme is proposed to quickly maintain frame length within an optimal length to reduce the framelength-adjusted frequency and to reduce power dissipation during identification process, and the identification efficiency of system can be maintained between 0.30 and 0.35. In [6], the scholar first use the tag estimation algorithm to estimate the number of tags, and then group the tags, which also greatly improve the efficiency of system identification, but because of the estimation algorithm to bring a large number of floating-point operations, resulting in excessive power consumption.

In view of the above problems, this paper proposed an improved scheme called Enhanced-Q algorithm to optimize $\mathrm{Q}$ algorithm. During the execution of the $\mathrm{Q}$ algorithm, the proposed algorithm embeds the BS algorithm into the Q algorithm, the collision tags are uniformly identified by the BS algorithm. The simulation results verify the effectiveness of the proposed algorithm.

\section{Q ALGORITHM ANALYSIS}

\section{A. DFSA Algorithm}

DFSA(Dynamic Frame Slotted ALOHA) algorithm is based on TDMA(Time Division Multiple Access) technology. The DFSA algorithm organizes several timeslots into one frame. The reader first broadcasts the length of the frame to all tags. Each tag then randomly selects a time slot to respond to the reader. If multiple tags select the same time slot, the data of the tags will conflict with each other, resulting in the reader cannot correctly identify the tags. The collision tags will wait for the next frame to communicate with the interrogator again. In each round of interrogation, the reader will dynamically adjust the frame length according to the number of tags. The reader will repeat this step until all tags have been correctly read or no collisions have occurred. Based on this, there are three cases for each slot in the frame: (1) Empty Slot. There is no tag to select the slot; (2) Single slot. There is only one tag to select the slot; (3) Collision slot. Two or more tags select the time slot. Figure I shows the process of the DFSA algorithm.

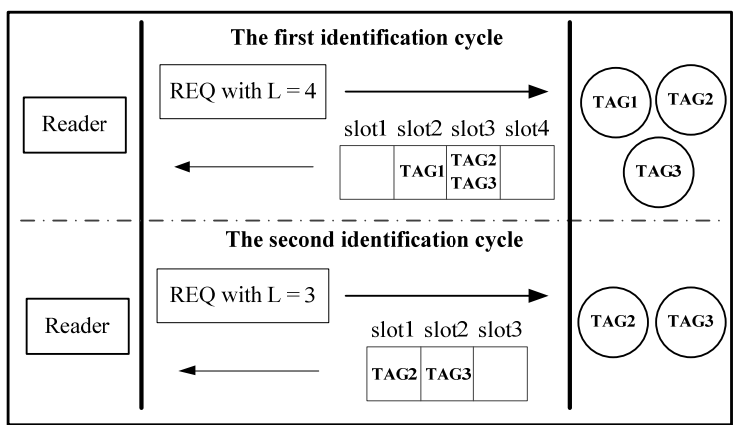

FIGURE I. PROCESS OF DFSA

Assuming that $L$ indicates the length of frame, $n$ indicates the quantity of tags. The distribution of tags in the time slot is approximately obeyed binomial distribution ${ }^{[7]}$. The probability that there are $r$ tags in a given time slot is:

$$
\mathrm{B}\left(r, n, \frac{1}{L}\right)=\left(\begin{array}{l}
n \\
r
\end{array}\right)\left(\frac{1}{L}\right)^{r}\left(1-\frac{1}{L}\right)^{n-r}
$$

Then the expected value $a_{l}$ of a single slot number is: 


$$
a_{1}=n\left(1-\frac{1}{L}\right)^{n-1}
$$

We define the system identification efficiency as the ratio of the number of single slots to the total number of slots in a single identification cycle, denoted by $E$.

$$
E=\frac{a_{1}}{L}=\frac{n}{L}\left(1-\frac{1}{L}\right)^{n-1}
$$

Derive Equation 3 and let the derivative be equal to zero, we will get:

$$
\frac{d E}{d n}=\left(1-\frac{1}{L}\right)^{n-1}+n\left(1-\frac{1}{L}\right)^{n-1} \ln \left(1-\frac{1}{L}\right)=0
$$

Using the limit theorem and Taylor series on the formula 4 solution, can be approximated:

$$
L \approx n
$$

From equation 5 it can be concluded that when the frame length and the number of tags are equal, the system identification efficiency can be maximized, and then the maximum value can be obtained according to equation 3 is 0.368

\section{B. Tag estimation}

Estimating the number of residual tags in the system is the key to DFSA algorithm to obtain the ideal identification efficiency of system. In recent years, there have been many researches on the estimation of the number of tags. The main idea is to observe the number of empty slots $E$, the number of single slots $S$, and the number of collision slots $C$ in the previous frame, and according to the statistical laws to achieve an estimate value of the number of tags.

Vogt ${ }^{[8]}$ takes the every time slot as the premise of the binomial distribution, using equation 1 to obtain the expected value of the empty slot $a_{0}=L^{*} \mathrm{~B}(0, n, 1 / L)$, the expected value of the single slot $a_{1}=L * \mathrm{~B}(1, n, 1 / L)$, and the expected value of the collision slot $a_{\geqslant 2}=L^{*} \mathrm{~B}(\geqslant 2, n, 1 / L)$. Vogt then uses equation 6 to compute the mean square error between the expected number of empty slots, the number of single slots, the number of collision slots and the observed value.

$$
\varepsilon_{v d}(n, E, S, C)=\min \left|\left(\begin{array}{c}
a_{0} \\
a_{l} \\
a_{\geq 2}
\end{array}\right)-\left(\begin{array}{c}
E \\
S \\
C
\end{array}\right)\right|
$$

Vogt points out that when the smallest $\varepsilon$ value is found, the corresponding value of $n$ is the estimated number of tags. Since the minimum number of tags is $n_{\text {min }}=S+2 C$, the search can be computed from $n_{\min }$.

Chen ${ }^{[9]}$ proposed a more accurate estimation algorithm.
At the end of a frame recognition cycle, Chen gives a posteriori probability model.

$$
P(n \mid E, S, C)=\frac{L !}{E ! S ! C !} p_{e}^{E} p_{s}^{S} p_{c}^{C}
$$

where $p_{e}=\mathrm{B}(0, n, 1 / L), p_{s}=\mathrm{B}(1, n, 1 / L)$ and

$$
p_{c}=\mathrm{B}(\geq 2, n, 1 / L) \text {. }
$$

The reader obtains the values of $\mathrm{E}, \mathrm{S}$, and $\mathrm{C}$, and calculates and finds the maximum value of expression 7 . The corresponding value of $\mathrm{n}$ is the estimated value of the number of tags.

It can be seen that most of the estimation algorithms estimate the number of tags by finding the maximum or minimum value of the formula, and improve the accuracy of the estimation by a large number of samples. This will introduce a large number of floating-point calculations, and increase total time for tag identification. To avoid complex computations, the EPC-C1G2 standard presents a simple frame length adaptive anti-collision Q algorithm.

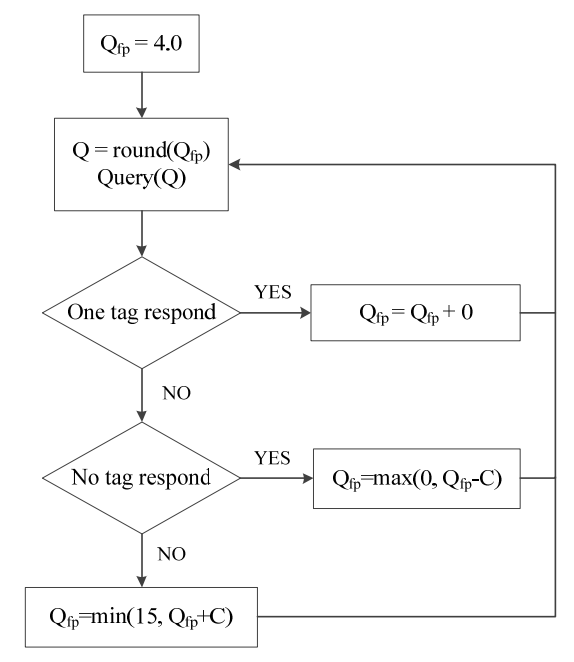

FIGURE II. FLOW CHART OF Q ALGORITHM

\section{Q Algorithm}

The Q algorithm consists of three basic instructions: Query, QueryRep and QueryAdjust. The reader first sends a Query command to initialize the identification cycle. The Query instruction contains a parameter $Q$ and sets the frame length to $2^{Q}$. Each tag itself maintains a timeslot counter. The tag receiving the Query command randomly selects a value in the range $\left[0,2^{Q}-1\right]$ and places the value in its slot counter, where the tags whose slot counter is 0 can respond to the reader. The reader then sends one or more QueryAdjust or QueryRep instructions depending on whether the $Q$ value needs to be changed. The QueryAdjust instruction adjusts the length of the frame by sending a new $Q$ value. The Tag received QueryAdjust instruction needs to reset its slot counter according to the latest $Q$ value. If tag do not need to update the $Q$ value, the reader will repeatedly send QueryRep 
instruction, the tag received QueryRep instruction will minus its slot counter by 1 , the tags whose timeslot counter equal to 0 will immediately respond to the reader. Figure II shows the flow chart of $\mathrm{Q}$ algorithm.

As shown in the figure above, the $\mathrm{Q}$ algorithm is an adaptive algorithm for frame length. When a frame has excess collision slots, a reader will end the frame early and broadcast a command with another larger frame length; when a frame has excess idle slots, a reader will also end the frame early and broad cast another less frame length. The method can be realized by parameters $Q$ and $Q_{f p}$. The parameter $Q$ is a positive integer in the range $[0,15]$, and the initial value of $Q$ is 4. $Q_{f p}$ is the floating point number of the $Q$ value. When the reader to detect the empty time slot or collision time slot occurs, it will use constant value of $C$ to adjust $Q_{f p}$. The value of $\mathrm{C}$ ranges from 0.1 to 0.5 . Function round $\left(Q_{f p}\right)$ indicates the rounded value of $Q_{f p}$.

\section{ENHANCED-Q ALGORITHM}

\section{A. BS Algorithm}

BS(Binary Splitting) ${ }^{[10]}$ algorithm is based on the binary deterministic RFID anti-collision algorithm, when the number of tags is smaller, the algorithm will achieve better performance and higher identification efficiency of system. The core idea of the algorithm is as follows: BS repeats splitting the collision tags into two subsets until each set has and only one tag. Each tag itself maintains a counter with an initial value of 0 , and only the tag with a counter of 0 responds to the reader. So at first all the tags will respond to the reader. The reader then sends a response message to the tag. The tag changes its counter value according to the reader's response. The conflicting tag will randomly select a binary number and add the binary number to the current counter. Such a set will be split into two subsets, as shown in Figure III.

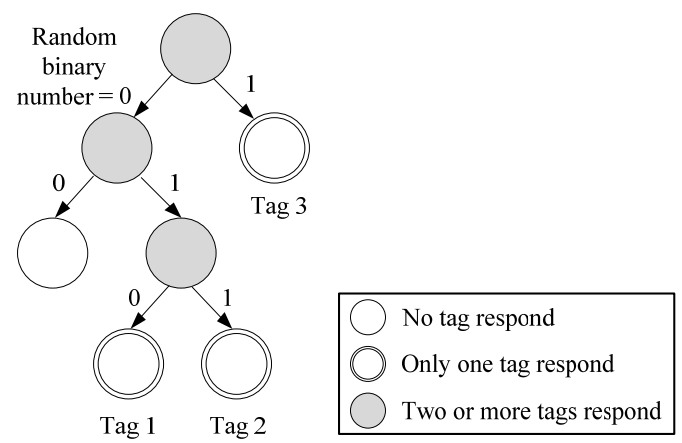

FIGURE III. TAG IDENTIFICATION OF BS

In the next round of identification cycles, if a collision occurs again, those tags whose counter is not zero will increment their counter, and those collision tags will repeat the above split operation. If no collision occurs, all tags decrement the counter, and for those whose counter value is decremented to zero, it will respond in the next round of interrogation.

\section{B. Enhanced-Q algorithm}

As the $\mathrm{Q}$ algorithm does not carry out the tag estimation, but through the constant $C$ to adjust the $Q$ value, which leads to the error between the frame length and the number of tags is relatively large, the system identification efficiency is not high. However, if the Q algorithm is optimized by the tag estimation algorithm, it will bring a huge floating point operation to the system and increase the total time of tag identification. In this paper, an improved Q algorithm is proposed and named as Enhanced-Q algorithm.

Figure IV gives a flowchart of Enhanced-Q algorithm, where function newFrameSize $(Q)$ implements that a new frame start and it frame length is $2^{Q}$. Actually, Enhanced-Q consists of Q algorithm and embedded BS, and its difference from $\mathrm{Q}$ algorithm is that when a current slot is collision and $\operatorname{round}\left(Q_{f p}\right)=Q$, collided tags in the slot will be resolved by BS algorithm at once. Only when all collided tags in the slot are resolved successfully, tags in the next slot will be read. As a result, a higher value of efficiency can be achieved in this manner.

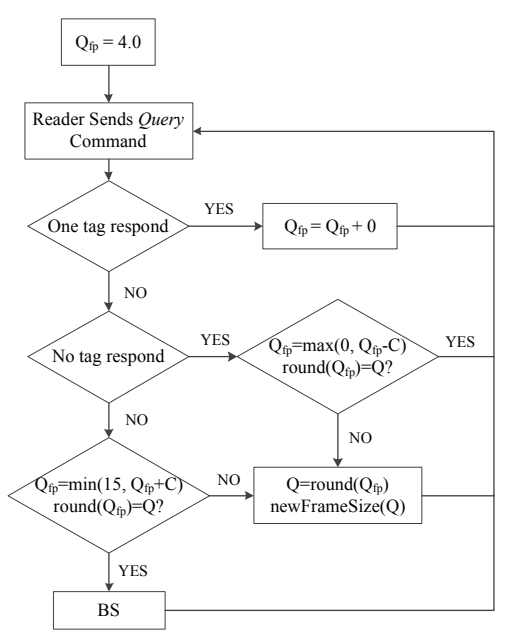

FIGURE IV. FLOW CHART OF ENHANCED-Q ALGORITHM

\section{SIMULATION EXPERIMENT AND ANALYSIS}

We evaluate the performance of the proposed algorithm by computer simulations. We individually perform each simulation 500 times, and average 500 simulation results into the final results.

Figure $\mathrm{V}$ presents the system identification efficiencies of Enhanced-Q algorithm, Q algorithm and DFSA algorithm, with the number of tags ranging from 100 to 4000 . In Enhanced-Q and $\mathrm{Q}$ algorithm, an initial value of $Q$ selects 4.0, and step $\mathrm{C}$ selects values as follows: if $0 \leq Q \leq 2, C=0.5$;if $Q \geq 10, C=0.1$;else, $C=1 / Q$. The initial frame length of DFSA will select 128 , and the estimate for number of tags in DFSA adopts the same method as in [7].

From Figure $\mathrm{V}$, when the number of tags increases from 100 to 4000 , Enhanced-Q algorithm has the highest value of system identification efficiency, around 0.4 and its efficiency is not affected by the number of tags increasing or decreasing. 
And the system identification efficiency curve of $Q$ algorithm is nearly horizontal at around 0.34 . We can find that the system identification efficiency of Ehanced-Q is 6\% higher than $\mathrm{Q}$ algorithm. When the number of tags range from 100 to 500, DFSA algorithm is more efficient than the $\mathrm{Q}$ algorithm. However, when the number of tags is greater than 500, the efficiency of the DFSA algorithm is lower than that of the $\mathrm{Q}$ algorithm due to the error caused by the tag estimation algorithm.

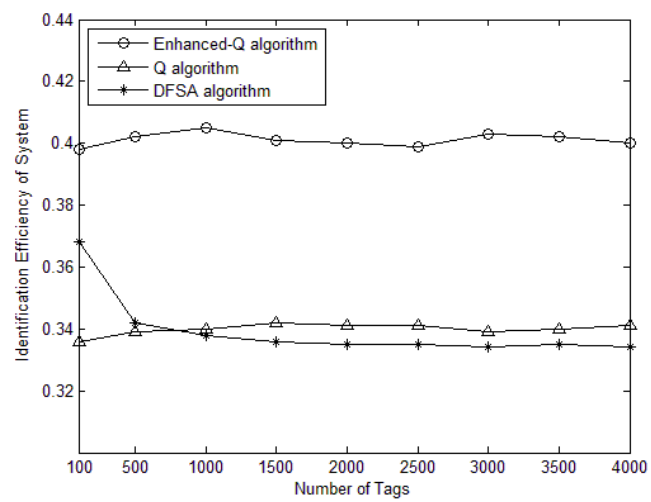

FIGURE V. IDENTIFICATION EFFICIENCY OF SYSTEM

Figure VI and VII give number of slots for identifying all tags when the number of tags ranging from 100 to 4000

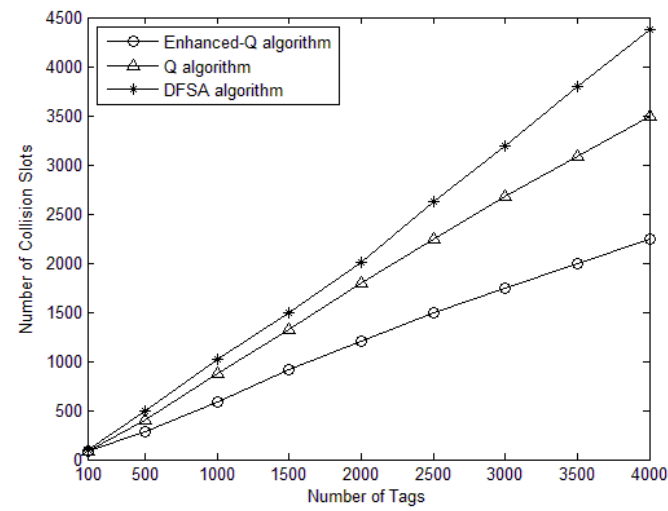

FIGURE VI. NUMBER OF COLLISION SLOTS

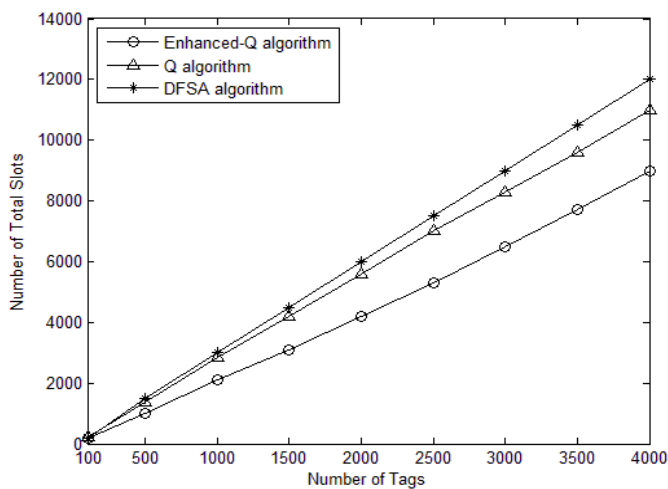

FIGURE VII. NUMBER OF TOTAL SLOTS
Similar to the results for the system identification efficiency, Enhanced-Q has better performance on identifying slot number than the other protocols. Figure VI give the number of collision slots for identifying all tags, and Figure VII give the number of total slots for identifying all tags. From the figures above, we can find that our proposed algorithm has the least number of collision slots and total slots. In terms of slot utilization, Enhanced-Q algorithm is higher than DFSA and Q algorithm.

\section{CONCLUSION}

When an RFID system identifies multiple tags, tag collisions will happen. The RFID system generally applies a tag anti-collision protocol to resolve the multi-tag collisions. Considering the low efficiency of Q algorithm, Enhanced-Q algorithm is proposed to optimize Q algorithm in this paper. The proposed algorithm does not use tag estimation algorithm to improve the identification efficiency of system. Instead, it uses the advantages of $\mathrm{Q}$ algorithm and $\mathrm{BS}$ algorithm, embeds BS algorithm into Q algorithm. Simulation results show that the new algorithm can effectively reduce the number of collision slots and total slots in the identification process, thus greatly improving the identification efficiency of system.

\section{REFERENCES}

[1] ZHOU Yancong, DONG Yongfeng, ZHANG Jin and GU Junhua "Anti-collision algorithm of dynamic frame slotted ALOHA based on hash grouping," Computer Engineering And Design. China, vol 37, pp. 540-544, Feb., 2016.

[2] H. Wu, Y. Zeng, J. Feng and Y. Gu, "Binary Tree Slotted ALOHA for Passive RFID Tag Anticollision," IEEE Transactions on Parallel and Distributed Systems, vol. 24, no. 1, pp. 19-31, Jan. 2013.

[3] EPCglobal Inc. "EPCTM radio-frequency identity protocols class-1 gen-2 UHF RFID protocol for communications at $860 \mathrm{MHz}-960 \mathrm{MHz}$ version 1.2.0,". Lawrenceville: EPCglobal Inc, 2010.

[4] C. Wang, M. Daneshmand, K. Sohraby and B. Li, "Performance analysis of RFID Generation-2 protocol," in IEEE Transactions on Wireless Communications, vol. 8, no. 5, pp. 2592-2601, May 2009.

[5] HAN Zhenwei, SONG Kefei, "Improved anti-collision Q-algorithm for RFID system," Computer Engineering and Design, vol. 32, no.7 pp.2314-2318, 2014

[6] Y. Maguire and R. Pappu, "An Optimal Q-Algorithm for the ISO 18000-6C RFID Protocol," in IEEE Transactions on Automation Science and Engineering, vol. 6, no. 1, pp. 16-24, Jan., 2009.

[7] XIE Lei, YIN Yafeng, CHEN Xi, "RFID Data Management: Algorithms, Protocols and Performance Evaluation," Chinese Journal of Computer, vol.36, no.3, 2015.

[8] P. Šolić, J. Radić and N. Rožić, "Energy Efficient Tag Estimation Method for ALOHA-Based RFID Systems," IEEE Sensors Journal, vol.14, no.10, 2014.

[9] Chen W, "An Accurate Tag Estimate Method for Improving the Performance of an RFID anticollision Algorithm Based on Dynamic Frame Length ALOHA," IEEE Transactions on Automation Science and Engineering, vol.6, no.1, 2009.

[10] Porta, Thomas F. La, Maselli and Gaia, "Anticollision Protocols for Single-Reader RFID Systems: Temporal Analysis and Optimization," IEEE Transactions on Mobile Computing, vol.10, no.2, 2011. 